\title{
Decoupling Capacitors for Multi-Voltage Power Distribution Systems
}

\author{
Mikhail Popovich and Eby G. Friedman, Fellow, IEEE
}

\begin{abstract}
Multiple power supply voltages are often used in modern high-performance ICs, such as microprocessors, to decrease power consumption without affecting circuit speed. To maintain the impedance of a power distribution system below a specified level, multiple decoupling capacitors are placed at different levels of the power grid hierarchy. The system of decoupling capacitors used in power distribution systems with multiple power supplies is described in this paper. The noise at one power supply can propagate to the other power supply, causing power and signal integrity problems in the overall system. With the introduction of a second power supply, therefore, the interaction between the two power distribution networks should be considered.

The dependence of the impedance and magnitude of the voltage transfer function on the parameters of the power distribution system is investigated. An antiresonance phenomenon is intuitively explained in this paper. It is shown that the magnitude of the voltage transfer function is strongly dependent on the parasitic inductance of the decoupling capacitors, decreasing with smaller inductance. Design techniques to cancel and shift antiresonant spikes out of range of the operating frequencies are presented. It is also shown that it is highly desirable to maintain the effective series inductance of the decoupling capacitors as low as possible to decrease the overshoots of the response of the dual-voltage power distribution system over a wide range of operating frequencies. A criterion for an overshoot-free voltage response is presented in this paper. It is noted that the frequency range of the overshoot-free voltage response can be traded off with the magnitude of the response.
\end{abstract}

Index Terms-Decoupling capacitors, multiple power supply voltages, power distribution networks, resistance-inductance-capacitance $(R L C)$ impedance.

\section{INTRODUCTION}

$\mathbf{P}$ OWER dissipation has become a critical design issue in high-performance microprocessors, as well as battery powered or wireless electronics, multimedia and digital signal processors, and high-speed networking. The most effective way to reduce power consumption is to lower the supply voltage. Reducing the supply voltage, however, increases the circuit delay [1]-[3]. The increased delay can be compensated by changing

Manuscript received November 4, 2004; revised January 10, 2006. This work was supported in part by the Semiconductor Research Corporation under Contract 2003-TJ-1068 and 2004-TJ-1207, the National Science Foundation under Contract CCR-0304574, the Fulbright Program under Grant 87481764, a grant from the New York State Office of Science, Technology, and Academic Research to the Center for Advanced Technology in Electronic Imaging Systems, and by grants from the Intel Corporation, the Eastman Kodak Company, Manhattan Routing, and the Intrinsix Corporation.

The authors are with the Department of Electrical and Computer Engineering, University of Rochester, Rochester, NY 14627 USA (e-mail: nhlover@ece.rochester.edu; friedman@ece.rochester.edu).

Digital Object Identifier 10.1109/TVLSI.2006.871756 the critical paths with behavioral transformations such as parallelization or pipelining [4]. The resulting circuit consumes less power while satisfying global throughput constraints at the cost of increased circuit area.

Recently, the use of multiple on-chip supply voltages has become common practice [5]. This strategy has the advantage of allowing modules along the critical paths to operate with the highest available voltage level (in order to satisfy target timing constraints) while permitting modules along the noncritical paths to use a lower voltage (thereby reducing the energy consumption). A multi-voltage scheme lowers the speed of those circuits operating at a lower power supply voltage without affecting the overall frequency, thereby reducing power without lowering the system frequency. In this manner, the energy consumption is decreased without affecting the circuit speed. This scheme results in a smaller area as compared to parallel architectures. The problem of using multiple supply voltages for reducing the power requirements has been investigated in the area of high-level synthesis for low-power [6], [7]. While it is possible to provide many supply voltages, in practice such a scenario is expensive. Practically, the availability of a small number of voltage supplies (two or three) is reasonable.

The design of the power distribution system has become an increasingly difficult challenge in modern CMOS circuits [8]. As CMOS technologies are scaled, the power supply voltage is lowered. As clock rates rise and more functions are integrated on-chip, the power consumed has greatly increased. Assuming that only a small percent of the power supply voltage (about 5\%) is permitted as ripple voltage (noise), a target impedance for an example power distribution system is [9]

$$
Z_{\text {target }}=\frac{V_{\mathrm{dd}} \times r}{I}=\frac{1.8 \text { volts } \times 5 \%}{50 \text { amperes }} \approx 0.002 \mathrm{ohms}
$$

where $V_{\mathrm{dd}}$ is the power supply voltage, $r$ is the allowed ripple voltage, and $I$ is the current. With general scaling theory [10], the current $I$ is increasing and the power supply voltage is decreasing. The impedance of a power distribution system should, therefore, be decreased to satisfy power noise constraints. The target impedance of a power distribution system is falling at an alarming rate, a factor of five per computer generation [11]. The target impedance must be satisfied not only at DC, but also at all frequencies where current transients exist [12]. Several major components of a power delivery system are used to satisfy a target impedance over a broad frequency range. A voltage regulator module (VRM) is effective up to about $1 \mathrm{kHz}$. Bulk capacitors supply current and maintain a low-power distribution system impedance from $1 \mathrm{kHz}$ to $1 \mathrm{MHz}$. High frequency ceramic capacitors maintain the power distribution system 
impedance from $1 \mathrm{MHz}$ to several hundred MHz. On-chip decoupling capacitors can be effective above $100 \mathrm{MHz}$.

By introducing a second power supply, the power supplies become coupled through a decoupling capacitor effectively placed between the two power supply voltages. Assuming a power delivery system with dual power supplies and only a small percent of the power supply voltage is permitted as ripple voltage (noise), the following inequality for the magnitude of a voltage transfer function $K_{V}$ should be satisfied:

$$
\left|K_{V}\right| \leq \frac{r \times V_{\mathrm{dd} 1}}{V_{\mathrm{dd} 2}}
$$

where $V_{\mathrm{dd} 1}$ is a lower voltage power supply, $r$ is the allowed ripple voltage on a lower voltage power supply, and $V_{\mathrm{dd} 2}$ is a higher voltage power supply. Since the higher voltage power supply is applied to the high-speed paths, as for example a clock distribution network, $V_{\mathrm{dd} 2}$ can be noisy. To guarantee that noise from the higher voltage supply does not affect the quiet power supply, (2) should be satisfied. For typical values of the power supply voltages and allowed ripple voltage for a CMOS $0.18-\mu \mathrm{m}$ technology, $\left|K_{V}\right|$ is chosen to be less than or equal to 0.1 to effectively decouple a noisy power supply from a quiet power supply.

A power distribution network is a complex multi-level system. The design of a power distribution system with multiple supply voltages is the primary focus of this paper. The influence of a second supply voltage on the system of decoupling capacitors is investigated. Noise coupling among multiple power distribution systems is also discussed in this paper. A criterion for producing an overshoot-free voltage response is determined. It is shown that to satisfy a target specification in order to decouple multiple power supplies, it is necessary to maintain the magnitude of the voltage transfer function below 0.1 . In certain cases, it is difficult to satisfy this criterion over the entire range of operating frequencies. In such a scenario, the frequency range of an overshoot-free voltage response can be traded off with the magnitude of the response. Case studies are also presented in the paper to quantitatively illustrate the proposed methodology for designing a system of decoupling capacitors.

This paper is organized as follows. The impedance of a power distribution system with multiple supply voltages is described in Section II. A case study of the dependence of the impedance on the power distribution system parameters is presented in Section III. The voltage transfer function of a power distribution system with multiple supply voltages is discussed in Section IV. Case studies examining the dependence of the magnitude of the voltage transfer function on the parameters of the power distribution system are illustrated in Section V. Some specific conclusions are summarized in Section VI.

\section{ImPEDANCE OF A POWER Distribution System With MULTIPLE SUPPLY VOLTAGES}

The impedance of a power distribution network is an important issue in modern high-performance ICs such as microprocessors. The impedance should be maintained below a target

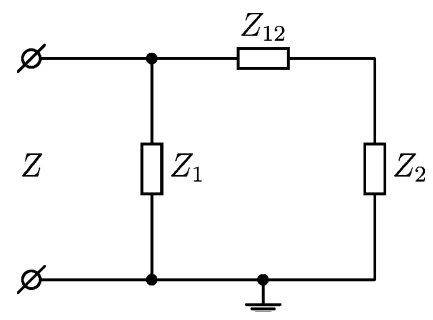

Fig. 1. Impedance of power distribution system with two supply voltages seen from the load of the power supply $V_{\mathrm{dd} 1}$.

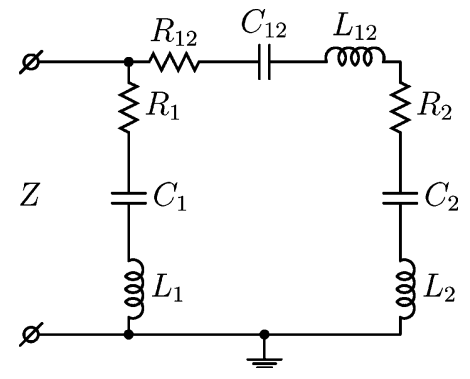

Fig. 2. Impedance of power distribution system with two supply voltages and the decoupling capacitors represented as series $R L C$ networks.

level to guarantee the power and signal integrity of a system. The impedance of a power distribution system with multiple power supplies is described in Section II-A. The antiresonance of capacitors connected in parallel is addressed in Section II-B. The dependence of the impedance on the power distribution system is investigated in Section II-C.

\section{A. Impedance of a Power Distribution System}

A model of the impedance of a power distribution system with two supply voltages is shown in Fig. 1. The impedance seen from the load of the power supply $V_{\mathrm{dd} 1}$ is illustrated. The model of the impedance is applicable for the load of the power supply $V_{\mathrm{dd} 2}$ if $Z_{1}$ is substituted for $Z_{2}$.

The impedance of the power distribution system shown in Fig. 1 can be modeled as

$$
Z=\frac{Z_{1} Z_{12}+Z_{1} Z_{2}}{Z_{1}+Z_{12}+Z_{2}}
$$

Decoupling capacitors have traditionally been modeled as a series resistance-inductance-capacitance $(R L C)$ network [13]. A schematic representation of a power distribution network with two supply voltages and the decoupling capacitors represented by $R L C$ series networks is shown in Fig. 2.

In this case, the impedance of the power distribution network is

$$
Z=\frac{a_{4} s^{4}+a_{3} s^{3}+a_{2} s^{2}+a_{1} s+a_{0}}{b_{3} s^{3}+b_{2} s^{2}+b_{1} s}
$$




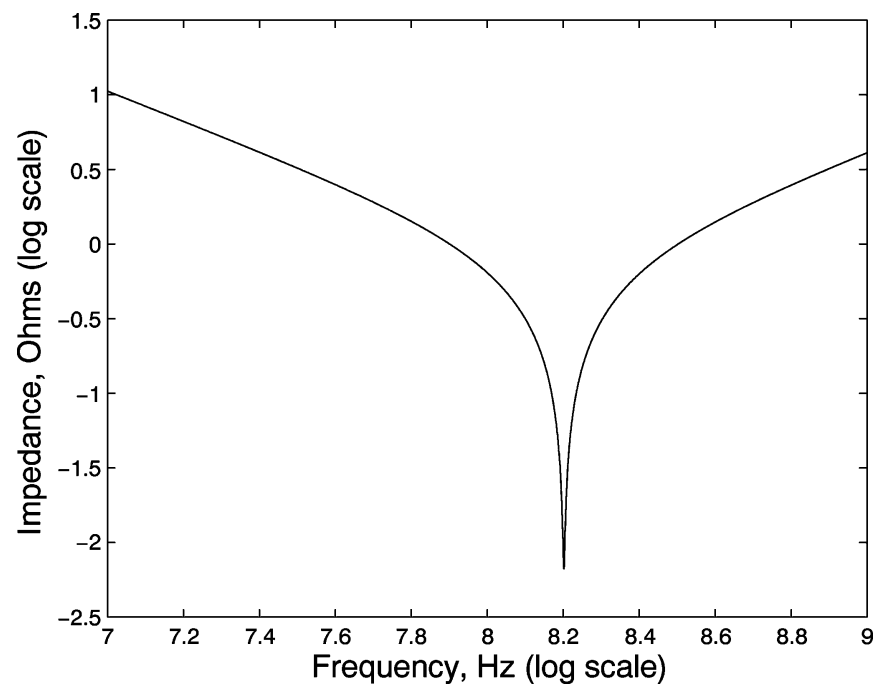

Fig. 3. Frequency dependence of the impedance of a power distribution system with dual supply voltages, $R_{1}=R_{12}=R_{2}=10 \mathrm{~m} \Omega, C_{1}=C_{12}=C_{2}=$ $1 \mathrm{nF}$, and $L_{1}=L_{12}=L_{2}=1 \mathrm{nH}$. Since all of the parameters of a power distribution system are identical, the system behaves as a single capacitor with a minimum at the resonant frequency. The minimum power distribution system impedance is limited by the ESR of the decoupling capacitors.

$$
\begin{aligned}
& \text { where } \\
& a_{4}=L_{1}\left(L_{12}+L_{2}\right) \\
& a_{3}=R_{1} L_{12}+R_{12} L_{1}+R_{1} L_{2}+R_{2} L_{1} \\
& a_{2}=R_{1} R_{12}+R_{1} R_{2}+\left(L_{1} / C_{12}\right)+\left(L_{12} / C_{1}\right)+\left(L_{1} / C_{2}\right) \\
& +\left(L_{2} / C_{1}\right) \\
& a_{1}=\left(R_{1} / C_{2}\right)+\left(R_{2} / C_{1}\right)+\left(R_{1} / C_{12}\right)+\left(R_{12} / C_{1}\right) \\
& a_{0}=\left(C_{12}+C_{2}\right) / C_{1} C_{12} C_{2} \\
& b_{3}=L_{1}+L_{12}+L_{2} \\
& b_{2}=R_{1}+R_{12}+R_{2} \\
& b_{1}=\left(1 / C_{1}\right)+\left(1 / C_{12}\right)+\left(1 / C_{2}\right)
\end{aligned}
$$$$
\text { and } s=j \omega \text { is a complex frequency. }
$$

The frequency dependence of the closed form expression for the impedance of a power distribution system with dual power supply voltages is illustrated in Fig. 3. The minimum power distribution system impedance is limited by the effective series resistance (ESR) of the decoupling capacitors. For on-chip applications, the ESR includes the parasitic resistance of the decoupling capacitor and the resistance of the power distribution network connecting a decoupling capacitor to a load. The resistance of the on-chip power distribution network is greater than the parasitic resistance of the on-chip decoupling capacitors. For on-chip applications, therefore, the ESR is represented by the resistance of the power delivery system. Conversely, for printed circuit board (PCB) applications, the resistance of the decoupling capacitors dominates the resistance of the power delivery system. In this case, therefore, the ESR is primarily the resistance of the decoupling capacitors. In order to achieve a target impedance as described by (1), multiple decoupling capacitors are placed at different levels of the power grid hierarchy [9].

As described in [14], the ESR of the decoupling capacitors does not change the location of the poles and zeros of the power distribution system impedance, only the damping factor of the $R L C$ system formed by the decoupling capacitor is affected. Representing a decoupling capacitor with a series $L C$ network,

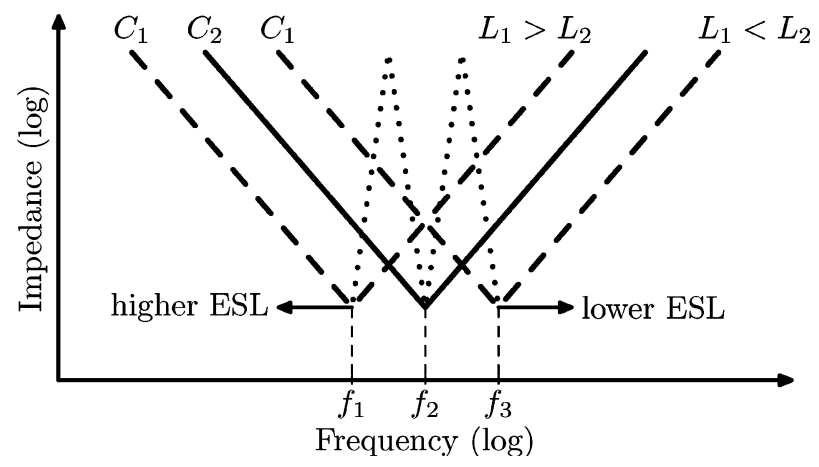

Fig. 4. Antiresonance of the two capacitors connected in parallel, $C_{2}=C_{1}$. Two antiresonant spikes appear between frequencies $f_{1}$ and $f_{2}$ and $f_{2}$ and $f_{3}$ (dotted lines).

the impedance of the power distribution system with dual power supply voltages is

$$
Z=\frac{a_{4} s^{4}+a_{2} s^{2}+a_{0}}{b_{3} s^{3}+b_{1} s}
$$

where

$$
\begin{aligned}
& a_{4}=L_{1}\left(L_{12}+L_{2}\right) \\
& a_{2}=\left(L_{1} / C_{12}\right)+\left(L_{12} / C_{1}\right)+\left(L_{1} / C_{2}\right)+\left(L_{2} / C_{1}\right) \\
& a_{0}=\left(C_{12}+C_{2}\right) / C_{1} C_{12} C_{2} \\
& b_{3}=L_{1}+L_{12}+L_{2} \\
& b_{1}=\left(1 / C_{1}\right)+\left(1 / C_{12}\right)+\left(1 / C_{2}\right) .
\end{aligned}
$$

\section{B. Antiresonance of Parallel Capacitors}

To maintain the impedance of a power distribution system below a specified level, multiple decoupling capacitors are placed in parallel at different levels of the power grid hierarchy. The ESR affects the quality factor of the $R L C$ system by acting as a damping element. The influence of the ESR on the impedance is, therefore, ignored. If all of the parameters of the circuit shown in Fig. 2 are equal, the impedance of the power distribution system can be described as a series $R L C$ circuit. Expression (5) has four zeros and three poles. Two zeros are located at the same frequency as the pole when all of the parameters of the circuit are equal. The pole is, therefore, canceled for this special case and the circuit behaves as a series $R L C$ circuit with one resonant frequency.

If the parameters of the power distribution system are not equal, the zeros of (5) are not paired. In this case, the pole is not canceled by a zero. For instance, in the case of two capacitors connected in parallel as shown in Fig. 4, in the frequency range from $f_{1}$ to $f_{2}$, the impedance of the capacitor $C_{1}$ has become inductive whereas the impedance of the capacitor $C_{2}$ remains capacitive. In this case, an $L C$ tank will produce a peak at a resonant frequency located between $f_{1}$ and $f_{2}$. Such a phenomenon is called antiresonance [9].

The location of the antiresonant spike depends on the ratio of the effective series inductance (ESL) of the decoupling capacitors. Depending upon the parasitic inductance, the peak impedance caused by the decoupling capacitor is shifted to a different frequency, as shown in Fig. 4. For instance, if the parasitic inductance of $C_{1}$ is greater than the parasitic inductance of $C_{2}$, the antiresonance will appear at a frequency 


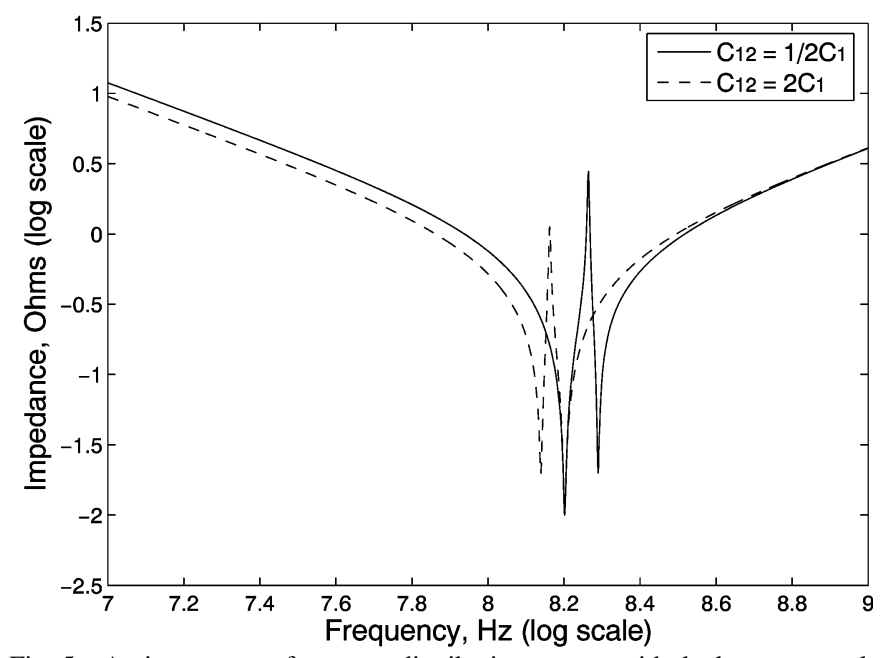

Fig. 5. Antiresonance of a power distribution system with dual power supply voltages, $R_{1}=R_{12}=R_{2}=10 \mathrm{~m} \Omega, C_{1}=C_{2}=1 \mathrm{nF}$, and $L_{1}=L_{12}=$ $L_{2}=1 \mathrm{nH}$. Depending upon the ratio of $C_{12}$ to $C_{1}$, the antiresonance appears before or after the resonant frequency of the system (the impedance minimum).

ranging from $f_{1}$ to $f_{2}$, i.e., before the self-resonant frequency $f_{2}$ of the capacitor $C_{2}$. If the parasitic inductance of $C_{1}$ is lower than the parasitic inductance of $C_{2}$, the antiresonance will appear at a frequency ranging from $f_{2}$ to $f_{3}$, i.e., after the self-resonant frequency of the capacitor $C_{2}$. The ESL of the decoupling capacitors, therefore, determines the frequency of the antiresonant spike of the system [15].

\section{Dependence of Impedance on Power Distribution System Parameters}

In practical applications, a capacitor $C_{12}$ placed between $V_{\mathrm{dd} 1}$ and $V_{\mathrm{dd} 2}$ exists either as a parasitic capacitance or as a decoupling capacitor. Intuitively, from Fig. 2, by decreasing the impedance $Z_{12}$ (increasing $C_{12}$ ), the greater part of $Z_{2}$ is connected in parallel with $Z_{1}$, reducing the impedance of the power distribution system as seen from the load of the power supply $V_{\mathrm{dd} 1}$. The value of a parasitic capacitance is typically much smaller than a decoupling capacitor such as $C_{1}$ and $C_{2}$. The decoupling capacitor $C_{12}$ can be chosen to be equal to or greater than $C_{1}$ and $C_{2}$. Depending upon the placement of the decoupling capacitors, the ESL can vary from $50 \mathrm{nH}$ at the power supply to almost negligible values on-chip. The ESL includes both the parasitic inductance of the decoupling capacitors and the inductance of the power delivery system. For on-chip applications, the inductance of the decoupling capacitors is much smaller than the inductance of the power distribution network and can be ignored. At the board level, however, the parasitic inductance of the decoupling capacitors dominates the overall inductance of a power delivery system. For these reasons, the model depicted in Fig. 2 is applicable to any hierarchical level of a power distribution system from the circuit board to on-chip.

Assuming $C_{1}=C_{2}$, if $C_{12}>C_{1}$, an antiresonance spike occurs at a lower frequency than the resonance frequency of an $R L C$ series circuit. If $C_{12}<C_{1}$, the antiresonance spike occurs at a higher frequency than the resonance frequency of an $R L C$ series circuit. This phenomenon is illustrated in Fig. 5.

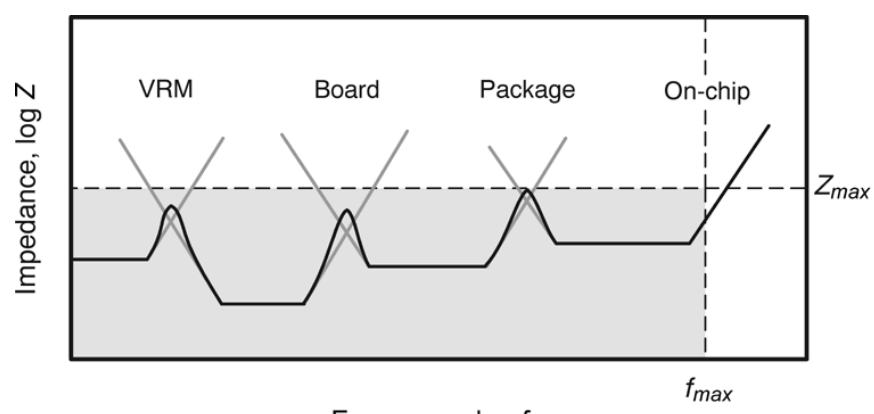

Frequency, $\log f$

Fig. 6. Impedance of the power distribution system as a function of frequency. Decoupling capacitors are placed at different hierarchical levels to shift an antiresonant spike above the maximum operating frequency of the system.

Antiresonance is highly undesirable because at a particular frequency, the impedance of a power distribution network can become unacceptably high. To cancel the antiresonance at a given frequency, a smaller decoupling capacitor is placed in parallel, shifting the antiresonance spike to a higher frequency. This procedure is repeated until the antiresonance spike appears at a frequency out of range of the operating frequencies of the system, as shown in Fig. 6.

Another technique for shifting the antiresonance spike to a higher frequency is to decrease the ESL of the decoupling capacitor. The dependence of the impedance of a power distribution system on the ESL is discussed next.

To determine the location of the antiresonant spikes, the roots of the denominator of (5) are evaluated. One pole is located at $\omega=0$. Two other poles are located at frequencies

$$
\omega= \pm \sqrt{\frac{C_{2}+\frac{C_{1} C_{2}}{C_{12}}+C_{1}}{C_{1} C_{2}\left(L_{1}+L_{12}+L_{2}\right)}}
$$

To shift the poles to a higher frequency, the ESL of the decoupling capacitors must be decreased. If the ESL of the decoupling capacitors is close to zero, the impedance of a power delivery network will not produce overshoots over a wide range of operating frequencies. Expression (6) shows that by minimizing the decoupling capacitor $C_{12}$ between the two supply voltages, the operating frequency of the overshoot-free impedance of a power delivery network can be increased.

The dependence of the power distribution system impedance on the ESL of $C_{12}$ is shown in Fig. 7(a). Note the strong dependence of the antiresonant frequency on the ESL of the decoupling capacitor located between $V_{\mathrm{dd} 1}$ and $V_{\mathrm{dd} 2}$. As discussed above, the location of the antiresonant spike is determined by the ESL ratio of the decoupling capacitors. The magnitude of the antiresonance spike is determined by the total ESL of $C_{1}$, $C_{12}$, and $C_{2}$, as shown in Fig. 7(b).

By lowering the system inductance, the quality factor is decreased. The peaks become wider in frequency and lower in magnitude. The amplitude of the antiresonant spikes can be decreased by lowering the ESL of all of the decoupling capacitors within the power distribution system. As shown in Fig. 7(b), decreasing the parasitic inductance of all of the decoupling capacitors of the system reduces the peak magnitude. When the parasitic inductance of $C_{12}$ is similar in magnitude to the other de- 


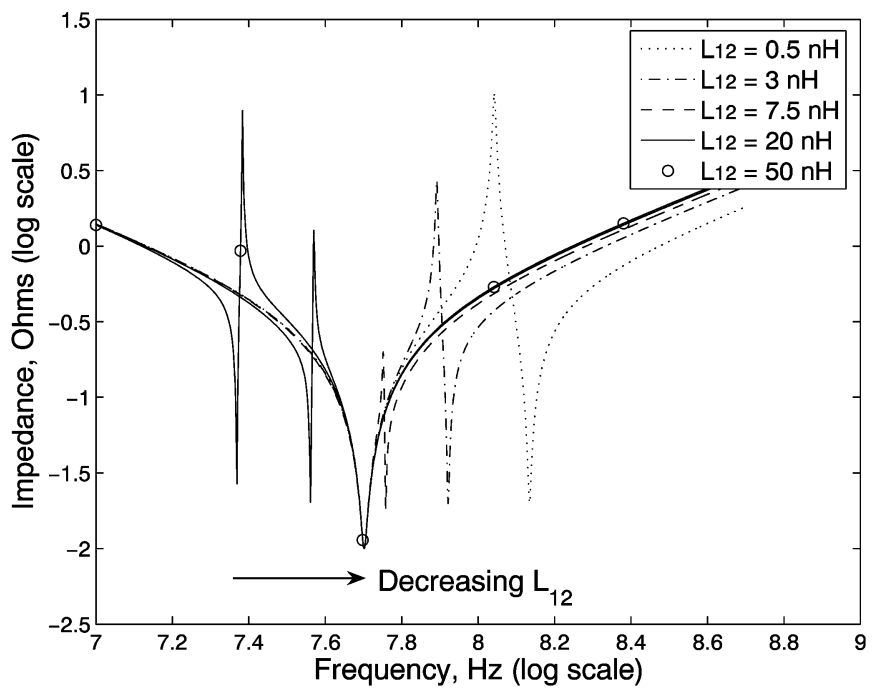

(a)

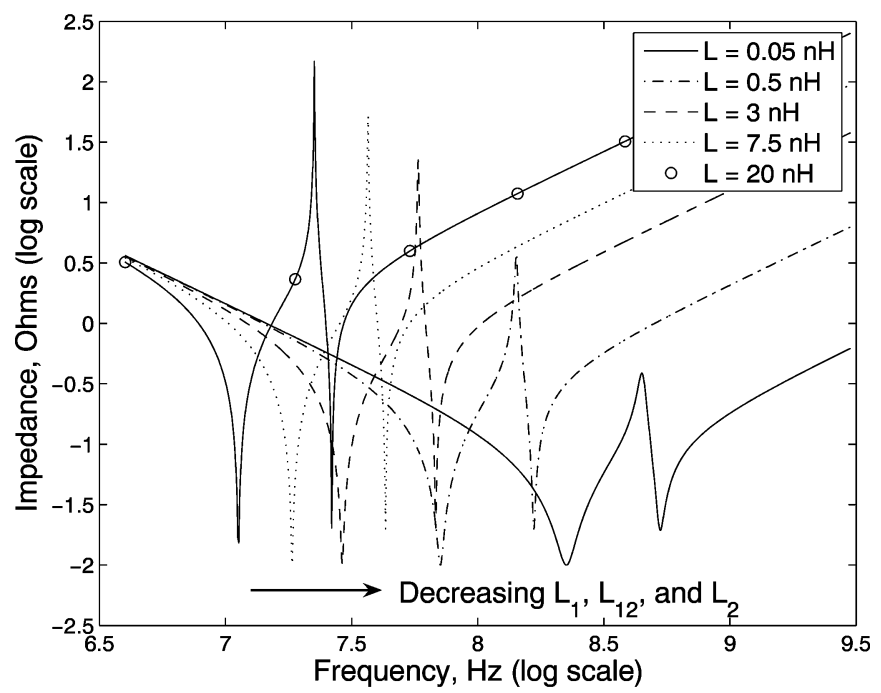

(b)

Fig. 7. Dependence of a dual $V_{\mathrm{dd}}$ power distribution system impedance on frequency for different ESL of the decoupling capacitors. The ESL of capacitors $C_{1}, C_{12}$, and $C_{2}$ is represented by $L_{1}, L_{12}$, and $L_{2}$, respectively. (a) By changing the ESL of the decoupling capacitor placed between the two power supplies, the ESL ratio of the capacitors changes and the antiresonant spike appears at different frequencies without decreasing the impedance of the power distribution system. $R_{1}=R_{12}=R_{2}=10 \mathrm{~m} \Omega, C_{1}=C_{2}=10 \mathrm{nF}$, $C_{12}=1 \mathrm{nF}$, and $L_{1}=L_{2}=1 \mathrm{nH}$. (b) The total ESL of a system should be decreased to lower both the impedance and the amplitude of the antiresonant spike. $R_{1}=R_{12}=R_{2}=10 \mathrm{~m} \Omega, C_{1}=C_{2}=10 \mathrm{nF}, C_{12}=1 \mathrm{nF}$, and $L_{1}=L_{12}=L_{2}=L$.

coupling capacitors, from (4), the poles and zeros do not cancel, affecting the circuit behavior. The zero at the resonant frequency of a system (the minimum value of the impedance) decreases the antiresonant spike. The closer the location of an antiresonant spike is to the resonant frequency of a system, the greater the influence of a zero on the antiresonance behavior. From a circuits perspective, the more similar the ESL of each capacitor, the smaller the amplitude of the antiresonant spike. Decreasing the inductance of the decoupling capacitors has the same effect as increasing the resistance. Increasing the parasitic resistance of a decoupling capacitor is limited by the target impedance of the power distribution system. Decreasing the inductance of a

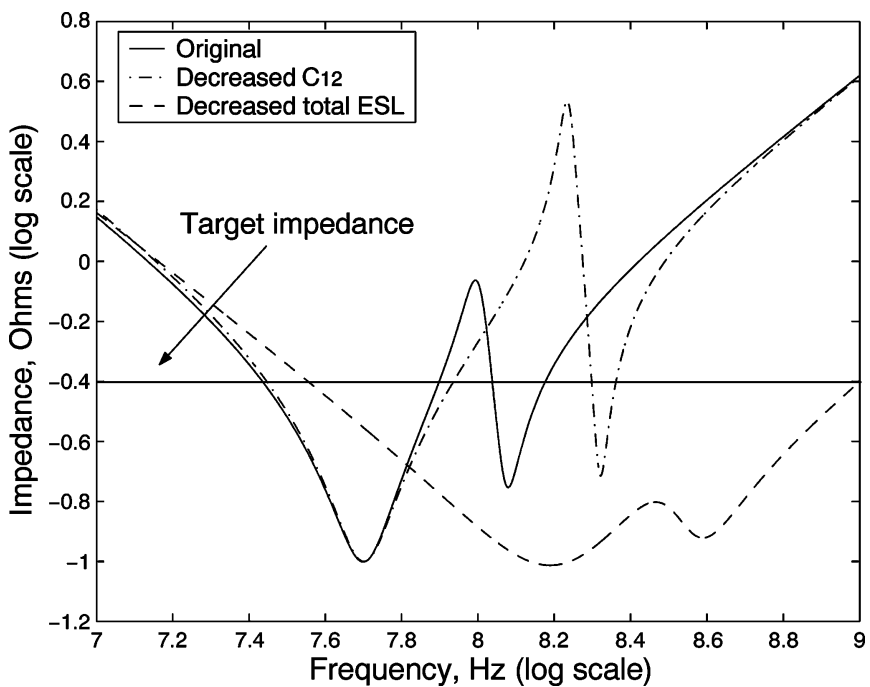

Fig. 8. Impedance of a power distribution system with dual power supply voltages as a function of frequency, $R_{1}=R_{12}=R_{2}=100 \mathrm{~m} \Omega, C_{1}=C_{2}=$ $10 \mathrm{nF}, C_{12}=1 \mathrm{nF}$, and $L_{1}=L_{12}=L_{2}=1 \mathrm{nH}$. The impedance of the example power distribution network produces an antiresonant spike with a magnitude greater than the target impedance (the solid line). The antiresonant spike is shifted to a higher frequency with a larger magnitude by decreasing $C_{12}$ to $0.3 \mathrm{nF}$ (the dashed-dotted line). By decreasing the total ESL of the system, the impedance can be maintained below the target impedance over a wide frequency range, from approximately $40 \mathrm{MHz}$ to $1 \mathrm{GHz}$ (the dashed line).

power distribution system is highly desirable and, if properly designed, the inductance of a power distribution system can be significantly reduced [16].

\section{Case Study of the Impedance of a Power DISTRIBUTION SYSTEM}

The dependence of the impedance on the power distribution system parameters is described in this section to quantitatively illustrate the concepts presented in Section II. An on-chip power distribution system is assumed in this example. The total budget of on-chip decoupling capacitance is distributed among the lowvoltage power supply $\left(C_{1}=10 \mathrm{nF}\right)$, high-voltage power supply $\left(C_{2}=10 \mathrm{nF}\right)$, and the capacitance placed between the two power supplies $\left(C_{12}=1 \mathrm{nF}\right)$. The ESR and ESL of the power distribution network are chosen to be equal to $0.1 \Omega$ and $1 \mathrm{nH}$, respectively. The target impedance is $0.4 \Omega$.

For typical values of an example power distribution system, an antiresonant spike is produced at approximately $100 \mathrm{MHz}$ with a magnitude greater than the target impedance, as shown in Fig. 8. According to (6), to shift the antiresonant spike to a higher frequency, the capacitor $C_{12}$ should be decreased. As $C_{12}$ is decreased to $0.3 \mathrm{nF}$, the antiresonant spike appears at a higher frequency, approximately $158 \mathrm{MHz}$, and is of higher magnitude. To further decrease the impedance of a power distribution system with multiple power supply voltages, the total ESL of the decoupling capacitors should be decreased. As the total ESL of the system is decreased to $0.1 \mathrm{nH}$, the impedance of the power distribution system is below the target impedance over a wide frequency range, from approximately $40 \mathrm{MHz}$ to $1 \mathrm{GHz}$. Three different tradeoff scenarios similar to the case study illustrated in Fig. 8 are summarized in Table I. The design parameters for each scenario represent typical values of board, package, and on-chip power distribution systems with decoupling capacitors, 
TABLE I

CASE StUdy OF THE IMPEDANCE OF a POWER DisTRIBUtion SyStem

\begin{tabular}{|c|c|c|c|c|}
\hline $\begin{array}{l}\text { Tradeoff } \\
\text { Scenario }\end{array}$ & $\begin{array}{c}\text { Power } \\
\text { Distribution } \\
\text { System } \\
\end{array}$ & $\begin{array}{l}\text { Minimum } \\
\text { frequency }\end{array}$ & $\begin{array}{l}\text { Maximum } \\
\text { frequency }\end{array}$ & $\begin{array}{l}\text { Frequency range } \\
\qquad \Delta f\end{array}$ \\
\hline I & $\begin{array}{c}\text { Original } \\
\text { Decreased } C_{12} \\
\text { Decreased } L_{1}, L_{12}, L_{2}\end{array}$ & $\begin{array}{l}4 \mathrm{kHz} \\
4 \mathrm{kHz} \\
4 \mathrm{kHz}\end{array}$ & $\begin{array}{c}35.48 \mathrm{kHz} \\
50.1 \mathrm{kHz} \\
1.26 \mathrm{MHz}\end{array}$ & $\begin{array}{c}31.48 \mathrm{kHz} \\
46.1 \mathrm{kHz} \\
1.256 \mathrm{MHz}\end{array}$ \\
\hline II & $\begin{array}{c}\text { Original } \\
\text { Decreased } C_{12} \\
\text { Decreased } L_{1}, L_{12}, L_{2}\end{array}$ & $\begin{array}{l}100 \mathrm{kHz} \\
100 \mathrm{kHz} \\
100 \mathrm{kHz}\end{array}$ & $\begin{array}{c}1 \mathrm{MHz} \\
2.82 \mathrm{MHz} \\
79 \mathrm{MHz}\end{array}$ & $\begin{array}{l}900 \mathrm{kHz} \\
2.72 \mathrm{MHz} \\
78.9 \mathrm{MHz}\end{array}$ \\
\hline III & $\begin{array}{c}\text { Original } \\
\text { Decreased } C_{12} \\
\text { Decreased } L_{1}, L_{12}, L_{2}\end{array}$ & $\begin{array}{l}560 \mathrm{MHz} \\
560 \mathrm{MHz} \\
890 \mathrm{MHz} \\
\end{array}$ & $\begin{array}{c}1 \mathrm{GHz} \\
1.12 \mathrm{GHz} \\
7.9 \mathrm{GHz} \\
\end{array}$ & $\begin{array}{l}440 \mathrm{MHz} \\
560 \mathrm{MHz} \\
7.01 \mathrm{GHz} \\
\end{array}$ \\
\hline $\begin{array}{l}\text { Scenario I } \\
\text { Board }\end{array}$ & \multirow{3}{*}{\multicolumn{4}{|c|}{$\begin{array}{l}\text { Original system: } R_{1}=R_{12}=R_{2}=1 \mathrm{~m} \Omega, L_{1}=L_{12}=L_{2}=50 \mathrm{nH}, C_{12}=100 \mu \mathrm{F}, C_{1}=C_{2}=1 \mathrm{mF} \\
\text { Decreased } C_{12}: R_{1}=R_{12}=R_{2}=1 \mathrm{~m} \Omega, L_{1}=L_{12}=L_{2}=50 \mathrm{nH}, C_{12}=20 \mu \mathrm{F}, C_{1}=C_{2}=1 \mathrm{mF} \\
\text { Decreased } L_{1}, L_{12}, L_{2}: R_{1}=R_{12}=R_{2}=1 \mathrm{~m} \Omega, L_{1}=L_{12}=L_{2}=5 \mathrm{nH}, C_{12}=100 \mu \mathrm{F}, C_{1}=C_{2}=1 \mathrm{mF} \\
\text { Original system: } R_{1}=R_{12}=R_{2}=1 \mathrm{~m} \Omega, L_{1}=L_{12}=L_{2}=1 \mathrm{nH}, C_{12}=3 \mu \mathrm{F}, C_{1}=C_{2}=50 \mu \mathrm{F} \\
\text { Decreased } C_{12}: R_{1}=R_{12}=R_{2}=1 \mathrm{~m} \Omega, L_{1}=L_{12}=L_{2}=1 \mathrm{nH}, C_{12}=1 \mu \mathrm{F}, C_{1}=C_{2}=50 \mu \mathrm{F} \\
\text { Decreased } L_{1}, L_{12}, L_{2}: R_{1}=R_{12}=R_{2}=1 \mathrm{~m} \Omega, L_{1}=L_{12}=L_{2}=100 \mathrm{pH}, C_{12}=3 \mu \mathrm{F}, C_{1}=C_{2}=50 \mu \mathrm{F} \\
\text { Original system: } R_{1}=R_{12}=R_{2}=10 \mathrm{~m} \Omega, L_{1}=L_{12}=L_{2}=10 \mathrm{pH}, C_{12}=1 \mathrm{nF}, C_{1}=C_{2}=4 \mathrm{nF} \\
\text { Decreased } C_{12}: R_{1}=R_{12}=R_{2}=10 \mathrm{~m} \Omega, L_{1}=L_{12}=L_{2}=10 \mathrm{pH}, C_{12}=0.3 \mathrm{nF}, C_{1}=C_{2}=4 \mathrm{nF} \\
\text { Decreased } L_{1}, L_{12}, L_{2}: R_{1}=R_{12}=R_{2}=10 \mathrm{~m} \Omega, L_{1}=L_{12}=L_{2}=1 \mathrm{pH}, C_{12}=1 \mathrm{nF}, C_{1}=C_{2}=4 \mathrm{nF}\end{array}$}} \\
\hline $\begin{array}{l}\text { Scenario II } \\
\text { Package }\end{array}$ & & & & \\
\hline $\begin{array}{l}\text { Scenario III } \\
\text { On-chip }\end{array}$ & & & & \\
\hline
\end{tabular}

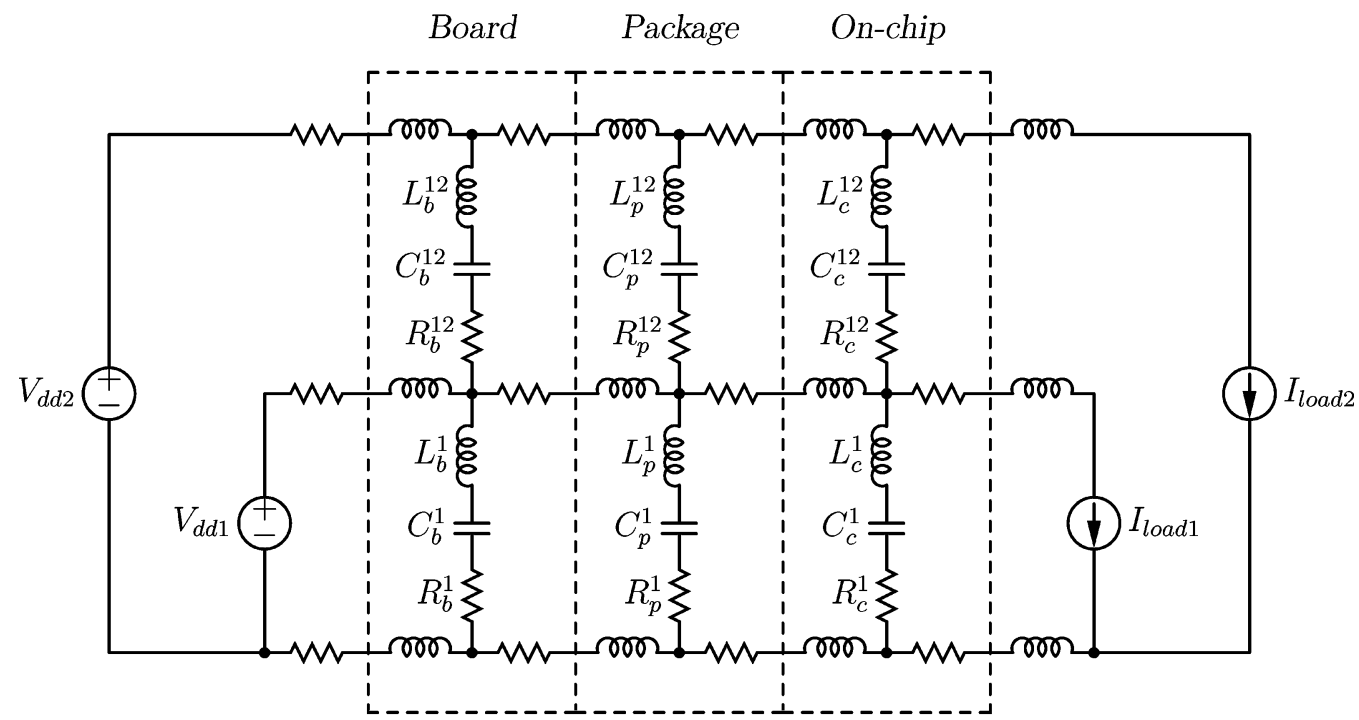

Fig. 9. Hierarchical model of a power distribution system with dual supply voltages and a single ground. The decoupling capacitors are represented by the series connected resistance, capacitance, and inductance. For simplicity, the decoupling capacitors placed between $V_{\mathrm{dd} 2}$ and ground are not illustrated. Subscripts $b$, $p$, and $c$ denote the board, package, and on-chip power delivery systems, respectively. Superscript 1 denotes the decoupling capacitors placed between $V_{\mathrm{dd} 1}$ and ground and superscript 12 denotes the decoupling capacitors placed between $V_{\mathrm{dd} 1}$ and $V_{\mathrm{dd} 2}$.

as shown in Fig. 9. The minimum and maximum frequencies denote the frequency range in which the impedance of a power delivery network seen from the load of $V_{\mathrm{dd} 1}$ does not exceed the target level of $400 \mathrm{~m} \Omega$. Note that by decreasing the decoupling capacitor placed between $V_{\mathrm{dd} 1}$ and $V_{\mathrm{dd} 2}$, the range of operating frequencies, where the target impedance is met, is slightly increased. Alternatively, if the total ESL of the system is lowered by an order of magnitude, the frequency range $\Delta f$ is increased by significantly more than an order of magnitude (for tradeoff scenario III, $\Delta f$ increases from $890 \mathrm{MHz}$ to $7.9 \mathrm{GHz}$ ).

The design of a power distribution system with multiple power supply voltages is a complex task and requires many iterative steps. In general, to maintain the impedance of a power delivery system below a target level, the proper combination of design parameters needs to be determined. In on-chip applica- tions, the ESL and $C_{12}$ can be chosen to satisfy specific values. At the board level, the ESR and $C_{12}$ can be adjusted to satisfy target impedance specifications. At the package level, the ESL, $C_{12}$, and ESR are the primary design parameters of the system. Usually, the total decoupling capacitance is constrained by the technology and application. In certain cases, it is possible to increase the decoupling capacitance. From (5), note that by increasing the decoupling capacitance, the overall impedance of a power distribution system with multiple power supply voltages can be significantly decreased.

\section{Voltage Transfer Function of POWER Distribution System With Multiple SupPly VolTAGES}

Classical methodologies for designing power distribution systems with a single power supply voltage typically only 


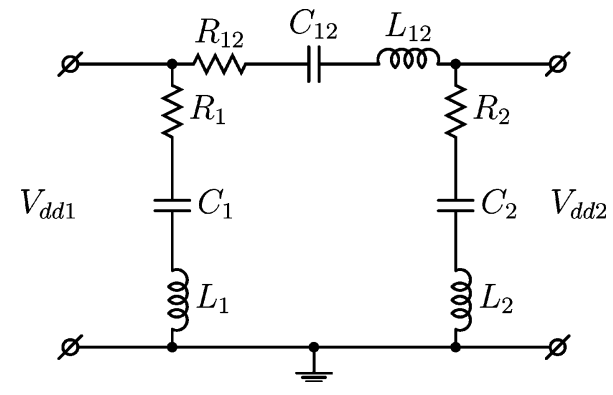

Fig. 10. Voltage transfer function of a power distribution network with two supply voltages and the decoupling capacitors represented as series $R L C$ networks.

consider the target output impedance of the network. By introducing a second power supply voltage, a decoupling capacitor is effectively placed between the two power supply voltages [17]. The problem of noise propagating from one power supply to the other power supply is aggravated if multiple power supply voltages are employed in a power distribution system. Since multiple power supplies are naturally coupled, the voltage transfer function of a multi-voltage power distribution network should be considered [18], [19]. The voltage transfer function of a power distribution system with dual power supplies is described in Section IV-A. The dependence of the magnitude of the voltage transfer function on certain parameters of the power distribution system is described in Section IV-B.

\section{A. Voltage Transfer Function of a Power Distribution System}

A power distribution system with two power supply voltages and the decoupling capacitors represented by an $R L C$ series network is shown in Fig. 10. All of the following formulae describing this system are symmetric in terms of the power supply voltages. The ESR and ESL of the three decoupling capacitors are represented by $R_{1}, R_{12}, R_{2}$, and $L_{1}, L_{12}, L_{2}$, respectively.

The voltage transfer function $K_{V}$ of a power distribution system with two power supply voltages and decoupling capacitors, represented by an $R L C$ network, is

$$
K_{V}=\frac{a_{2} s^{2}+a_{1} s+a_{0}}{b_{2} s^{2}+b_{1} s+b_{0}}
$$

where

$$
\begin{aligned}
& a_{2}=L_{2} C_{2} \\
& a_{1}=R_{2} C_{2} \\
& a_{0}=C_{12} \\
& b_{2}=C_{12} C_{2}\left(L_{12}+L_{2}\right) \\
& b_{1}=C_{12} C_{2}\left(R_{12}+R_{2}\right) \\
& b_{0}=C_{12}+C_{2} .
\end{aligned}
$$

Rearranging, (7) can be written as

$$
K_{V}=\frac{1}{\frac{a_{2} s^{2}+a_{1} s+a_{0}}{b_{2} s^{2}+b_{1} s+b_{0}}+1}
$$

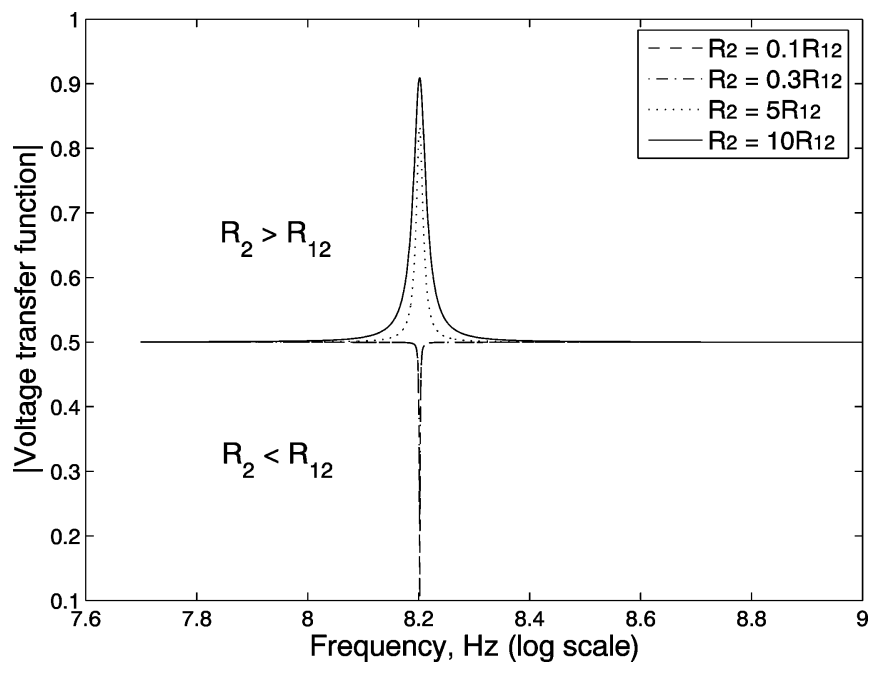

Fig. 11. Dependence of the magnitude of the voltage transfer function on frequency of a dual $V_{\mathrm{dd}}$ power distribution system for different values of ESR of the decoupling capacitors, $R_{12}=10 \mathrm{~m} \Omega, C_{12}=C_{2}=1 \mathrm{nF}$, and $L_{12}=$ $L_{2}=1 \mathrm{nH}$.

where

$$
\begin{aligned}
& a_{2}=L_{12} C_{12} C_{2} \\
& a_{1}=R_{12} C_{12} C_{2} \\
& a_{0}=C_{2} \\
& b_{2}=L_{2} C_{12} C_{2} \\
& b_{1}=R_{2} C_{12} C_{2} \\
& b_{0}=C_{12} .
\end{aligned}
$$

Equations (7) and (8) are valid only for nonzero frequency, i.e., for $s>0$. Note from (7), that if all of the parameters of a power distribution system are identical, the transfer function equals 0.5 and is independent of frequency. The dependence of the voltage transfer function on the parameters of the power distribution system is discussed below.

\section{B. Dependence of Voltage Transfer Function on Power Distribution System Parameters}

In power distribution systems with two supply voltages, the higher power supply is usually provided for the high-speed circuits, while the lower power supply is used in the noncritical paths [20]. The two power supplies are often strongly coupled, implying that voltage fluctuations on one power supply propagate to the other power supply. The magnitude of the voltage transfer function should be sufficiently small in order to decouple the noisy power supply from the quiet power supply. The objective is, therefore, to achieve a transfer function $K_{V}$ such that the two power supplies are effectively decoupled.

The dependence of the magnitude of the voltage transfer function on frequency for different values of the ESR of the power distribution network with decoupling capacitors is shown in Fig. 11. Reducing the ESR of a decoupling capacitor decreases the magnitude and range of the operating frequency of the transfer function. Note that to maintain $\left|K_{V}\right|$ below or equal to 0.5 , the following inequality has to be satisfied:

$$
R_{2} \leq R_{12}
$$




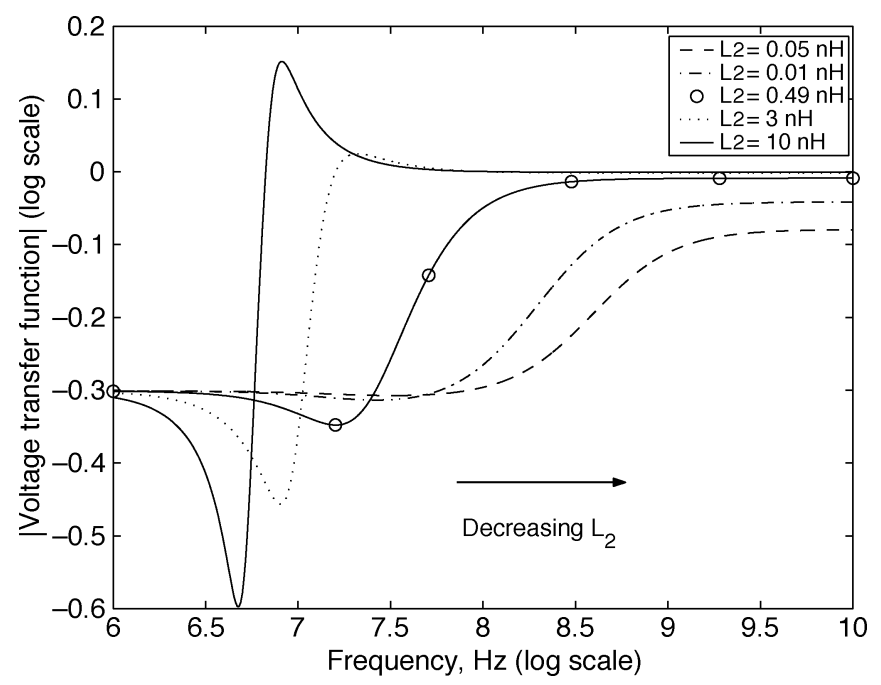

Fig. 12. Frequency dependence of the voltage transfer function of a dual $V_{\text {dd }}$ power distribution system for different values of ESL of the decoupling capacitors, $R_{12}=R_{2}=100 \mathrm{~m} \Omega, C_{12}=C_{2}=100 \mathrm{nF}$, and $L_{12}=10 \mathrm{pH}$.

This behavior can be explained as follows. From (8), to maintain $\left|K_{V}\right|$ below or equal to 0.5

$$
\frac{L_{12} C_{12} C_{2} s^{2}+R_{12} C_{12} C_{2} s+C_{2}}{L_{2} C_{12} C_{2} s^{2}+R_{2} C_{12} C_{2} s+C_{12}}+1 \geq 2 .
$$

For equal decoupling capacitors and parasitic inductances, (10) leads directly to (9). Generally, to maintain $\left|K_{V}\right|$ below or equal to 0.5

$$
\begin{aligned}
L_{12} C_{12} C_{2} s^{2}+R_{12} C_{12} C_{2} s+C_{2} & \\
& \geq L_{2} C_{12} C_{2} s^{2}+R_{2} C_{12} C_{2} s+C_{12}
\end{aligned}
$$

From (11), in order to maintain the magnitude of the voltage transfer function below or equal to 0.5, the ESR and ESL of the decoupling capacitors should be chosen to satisfy (11).

To investigate the dependence of the magnitude of the voltage transfer function on the decoupling capacitors and associated parasitic inductances, the roots of the characteristic equation, the denominator of (7), should be analyzed. To produce an overshoot-free response, the roots of the characteristic equation must be real, yielding

$$
R_{12}+R_{2} \geq 2 \sqrt{\frac{\left(L_{12}+L_{2}\right)\left(C_{12}+C_{2}\right)}{C_{12} C_{2}}} .
$$

In the case where $R_{12}=R_{2}=R, L_{12}=L_{2}=L$, and $C_{12}=C_{2}=C$, (12) reduces to the well-known formula [21]

$$
R \geq 2 \sqrt{\frac{L}{C}}
$$

The dependence of the magnitude of the voltage transfer function on the ESL of a power distribution system is shown in Fig. 12. For the power distribution system parameters listed in Fig. 12, the critical value of $L_{2}$ to ensure an overshoot-free response is $0.49 \mathrm{nH}$. Therefore, in order to produce an overshoot-free response, the ESL of $C_{2}$ should be smaller than or equal to $0.49 \mathrm{nH}$.

Intuitively, if the ESL of a system is large, the system is underdamped and produces an undershoot and an overshoot. By decreasing $L_{2}$, the resulting inductance of the system in (12) is lowered and the system becomes more damped. As a result, the undershoots and overshoots of the voltage response are significantly smaller. If $L_{2}$ is decreased to the critical value, the system becomes overdamped, producing an overshoot-free voltage response.

As shown in Fig. 12, the magnitude of the voltage transfer function is strongly dependent on the ESL, decreasing with smaller ESL. It is highly desirable to maintain the ESL as low as possible to achieve a small overshoot-free response characterizing a dual $V_{\text {dd }}$ power distribution system over a wide range of operating frequencies. Criterion (12) is strict and produces an overshoot-free voltage response. In most applications, if small overshoots (about 1\%) are permitted, (12) is less strict, permitting the parameters of a power distribution network to vary over a wider range.

For the parameters listed in Fig. 12, the minimum overshoot-free voltage response equals 0.5 . It is often necessary to maintain an extremely low magnitude voltage transfer function over a specific frequency range. This behavior can be achieved by varying one of the three design parameters (ESR, ESL, or $C$ ) characterizing a decoupling capacitor while maintaining the other parameters at predefined values. In this case, for different decoupling capacitors, the magnitude of the voltage transfer function is maintained as low as 0.1 over the frequency range from DC to the self-resonant frequency of the decoupling capacitor induced by the $R L C$ series circuit (hereafter, called the break frequency).

The inductance of the decoupling capacitor has an opposite effect on the magnitude of the voltage transfer function. By increasing the ESL of a dual $V_{\text {dd }}$ power distribution system, the magnitude of the voltage transfer function can be maintained below 0.1 from the self-resonant frequency (or break frequency) of the decoupling capacitor to the maximum operating frequency. From (8), for frequencies smaller than the break frequency, the magnitude of the voltage transfer function is approximately $C_{12} / C_{2}$. For frequencies greater than the break frequency, the magnitude of the voltage transfer function is approximately $L_{2} / L_{12}$. To maintain $\left|K_{V}\right|$ below 0.1 , it is difficult to satisfy (12), and the range of operating frequency is divided by the break frequency into two ranges. This phenomenon is illustrated in Fig. 13(a) and (b).

\section{Case Study of the Voltage Response of a Power DISTRIBUTION SYSTEM}

The dependence of the voltage transfer function on the parameters of a power distribution system is described in this section to quantitatively illustrate the concepts presented in Section IV. An on-chip power distribution system is assumed in this example. In modern high-performance ICs, the total on-chip decoupling capacitance can exceed $300 \mathrm{nF}$, occupying about $20 \%$ of the total area of an IC [22]. In this example, the on-chip decoupling capacitance is assumed to be $160 \mathrm{nF}$. The total budgeted 


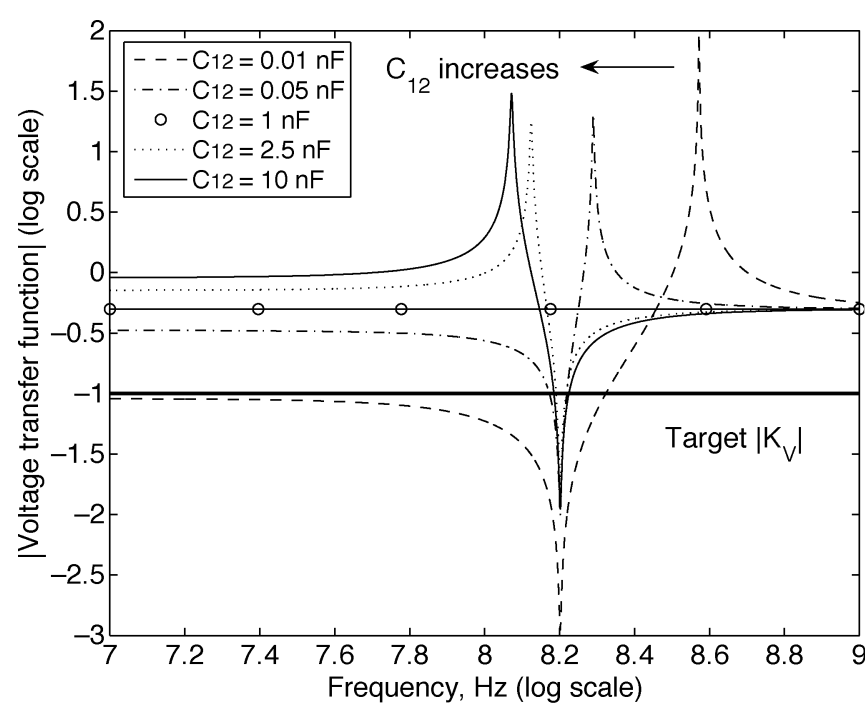

(a)

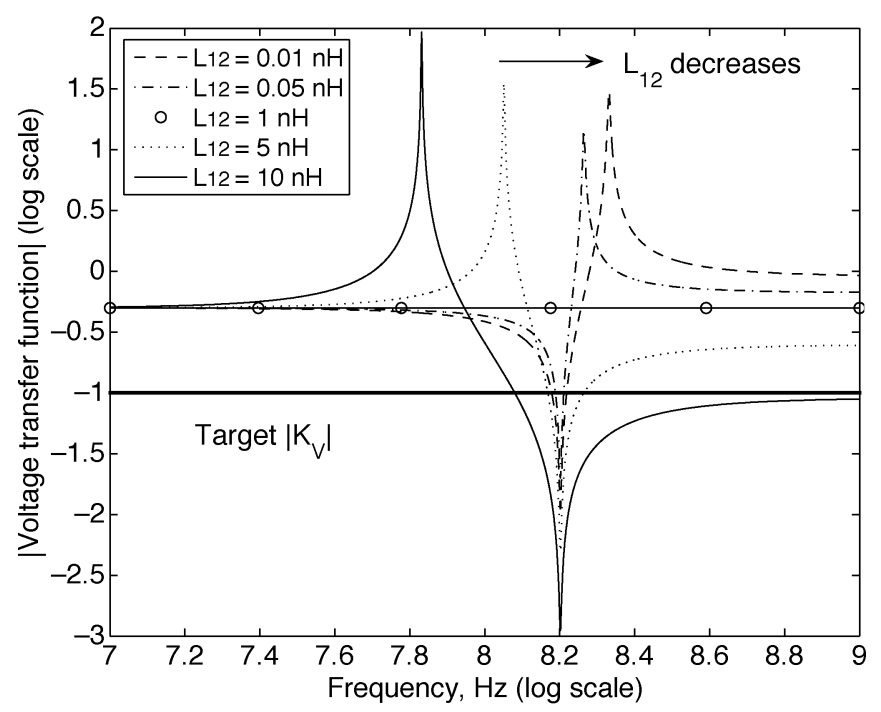

(b)

Fig. 13. Frequency dependence of the voltage transfer function of a dual $V_{\mathrm{dd}}$ power distribution system. The ESR and ESL of the decoupling capacitors for each power supply are represented by $R_{12}, R_{2}$ and $L_{12}, L_{2}$, respectively. (a) To maintain the voltage transfer function below 0.1 for frequencies smaller than the brake frequency, both the capacitor placed between the two power supplies and the ESL of all of the decoupling capacitors should be decreased. $R_{12}=R_{2}=$ $10 \mathrm{~m} \Omega, C_{2}=1 \mathrm{nF}$, and $L_{12}=L_{2}=1 \mathrm{nH}$. (b) To decouple the power supplies in frequencies ranging from the brake frequency to infinity, both the ESL of the capacitor placed between the two power supply voltages and the decoupling capacitors should be increased. $R_{12}=R_{2}=10 \mathrm{~m} \Omega, C_{12}=C_{2}=1 \mathrm{nF}$, and $L_{2}=1 \mathrm{nH}$.

on-chip decoupling capacitance is arbitrarily distributed among the low-voltage power supply $\left(C_{1}=100 \mathrm{nF}\right)$, high-voltage power supply $\left(C_{2}=40 \mathrm{nF}\right)$, and the capacitance placed between the two power supplies $\left(C_{12}=20 \mathrm{nF}\right)$. The ESR and ESL of the decoupling capacitor are chosen to be $0.1 \mathrm{ohms}$ and $1 \mathrm{nH}$, respectively.

In designing a power distribution system with dual power supply voltages, it is crucial to produce an overshoot-free voltage response over the range of operating frequencies. Depending on the system parameters, it can be necessary to

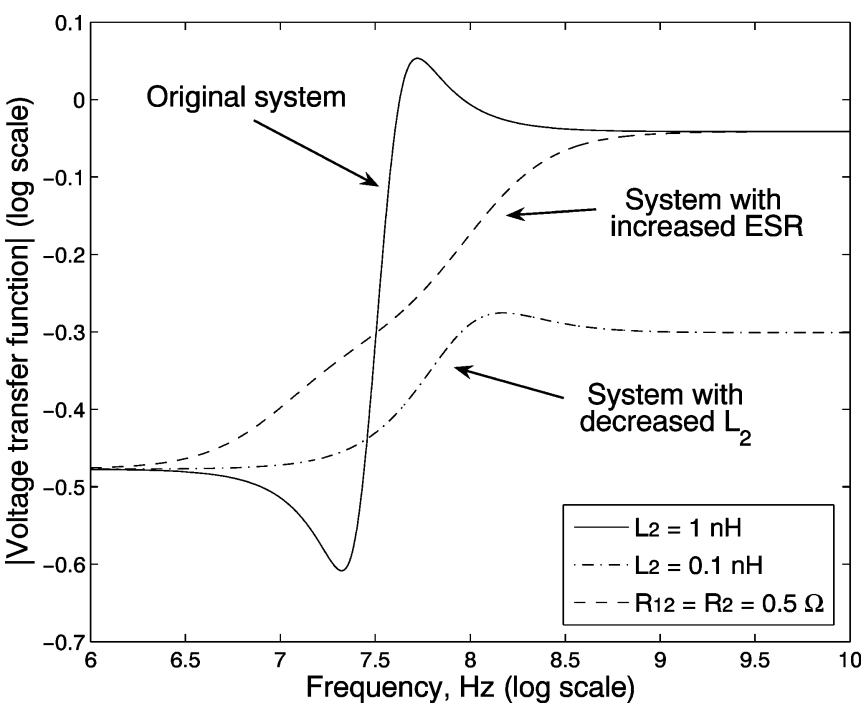

Fig. 14. Dependence of the magnitude of the voltage transfer function of a dual $V_{\mathrm{dd}}$ power distribution system on frequency for different values of the ESR and ESL of the decoupling capacitors, $R_{12}=R_{2}=0.1 \Omega, C_{12}=20 \mathrm{nF}, C_{2}=$ $40 \mathrm{nF}$, and $L_{12}=L_{2}=1 \mathrm{nH}$. The initial system with $L_{2}=1 \mathrm{nH}$ produces an overshoot (solid line). To produce an overshoot-free voltage response, either the ESR of the system should be increased (dashed line) or the ESL should be decreased (dash-dotted line).

further decouple the power supplies, requiring the magnitude of the voltage transfer function to be decreased. In this case, it is difficult to satisfy (12) and the range of operating frequencies is therefore divided into two. There are two possible scenarios: 1) the two power supplies should be decoupled as much as possible from DC to the break frequency and 2) the two power supplies should be decoupled as much as possible from the break frequency to infinity.

Note that infinite frequency is constrained by the maximum operating frequency of a specific system. Also note that the ESR, ESL, and magnitude of the decoupling capacitors can be considered as design parameters. The ESR is limited by the target impedance of the power distribution network. The ESL, however, can vary significantly. The total budgeted decoupling capacitance is distributed among $C_{1}, C_{12}$, and $C_{2}$. Note that $C_{12}$ can range from zero (no decoupling capacitance between the two power supplies) to $C_{12}=C_{\text {total }}-C_{1}-C_{2}$ (the maximum available decoupling capacitance between the two power supplies), where $C_{\text {total }}$ is the total budgeted decoupling capacitance.

\section{A. Overshoot-Free Magnitude of a Voltage Transfer Function}

For typical values of an example power distribution system, (12) is not satisfied and the response of the voltage transfer function produces an overshoot as shown in Fig. 14. To produce an overshoot-free voltage response, the capacitor placed between the two power supplies should be significantly increased, permitting the ESR and ESL to be varied. Increasing the ESR of the decoupling capacitors to 0.5 ohms produces an overshoot-free response. By decreasing the ESL of $C_{2}$, the overshoot-free voltage response can be further decreased, also shown in Fig. 14. As described in Section IV-B, at low frequency the magnitude of the voltage transfer function is 
approximately $C_{12} / C_{2}$. Note that all curves start from the same point. By increasing the ESR, the system becomes overdamped and produces an overshoot-free voltage response. Since the ESR does not change the $L_{2} / L_{12}$ ratio, the voltage response of the overdamped system is the same as the voltage response of the initial underdamped system. Note that the dashed line and solid line converge to the same point at high frequencies, where the magnitude of the voltage transfer function is approximately $L_{2} / L_{12}$. By decreasing $L_{2}$, the total ESL of the system is lowered and the system becomes overdamped, producing an overshoot-free voltage response. Also, since the $L_{2} / L_{12}$ ratio is lowered, the magnitude of the voltage response is significantly reduced at high frequencies.

In general, a design methodology for producing an overshootfree response of a power distribution system with dual power supply voltages is as follows. Based on the available decoupling capacitance for each power supply, the value of the decoupling capacitor placed between the two power supplies is determined by $C_{12}=C_{\text {total }}-C_{1}-C_{2}$. The ESR is chosen to be less than or equal to the target impedance to satisfy the impedance constraint. The critical ESL of the capacitors $C_{12}$ and $C_{2}$ is determined from (12). If the parasitic inductance of $C_{12}$ and $C_{2}$ is less than or equal to the critical ESL, the system will produce an overshoot-free voltage response and no adjustment is required. Otherwise, the total decoupling capacitance budget should be redistributed among $C_{1}, C_{12}$, and $C_{2}$ until (12) is satisfied. In certain cases, the total budgeted decoupling capacitance should be increased to satisfy (12).

\section{B. Tradeoff Between the Magnitude and Frequency Range}

If it is necessary to further decouple the power supplies, the frequency range of the overshoot-free voltage response can be traded off with the magnitude of the voltage response, as described in Section IV-B. There are two ranges of interest. The magnitude of the voltage transfer function can be decreased over the frequency range from DC to the break frequency or from the break frequency to the highest operating frequency. For the example power distribution system, as shown in Fig. 15(a), the magnitude of the voltage transfer function is overshoot-free from the break frequency to the highest operating frequency. To further decrease the magnitude of the voltage transfer function over a specified frequency range, the ESL of the decoupling capacitor placed between the two power supply voltages should be increased and $C_{12}$ should be the maximum available decoupling capacitance, $C_{12}=C_{\text {total }}-C_{1}-C_{2}$.

To decrease the magnitude of the voltage transfer function of a power distribution system with dual power supply voltages for frequencies less than the break frequency, the ESL of all of the decoupling capacitors and the value of $C_{12}$ should be decreased, as shown in Fig. 15(b). If it is necessary to completely decouple the two power supply voltages, $C_{12}$ should be minimized. This behavior can be explained as follows. The initial system produces an overshoot-free voltage response in the frequency range from $D C$ to the highest operating frequency of the system. In order to satisfy the target $\left|K_{V}\right|$ at high frequencies, $L_{12}$ should be increased in order to decrease the $L_{2} / L_{12}$ ratio. By increasing $L_{12}$, the magnitude of the voltage response falls

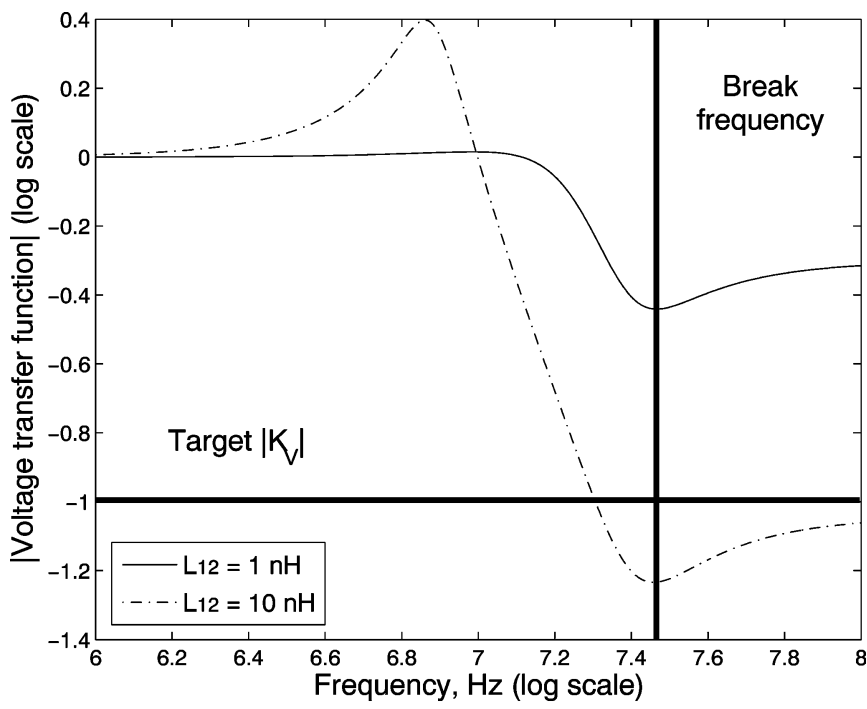

(a)

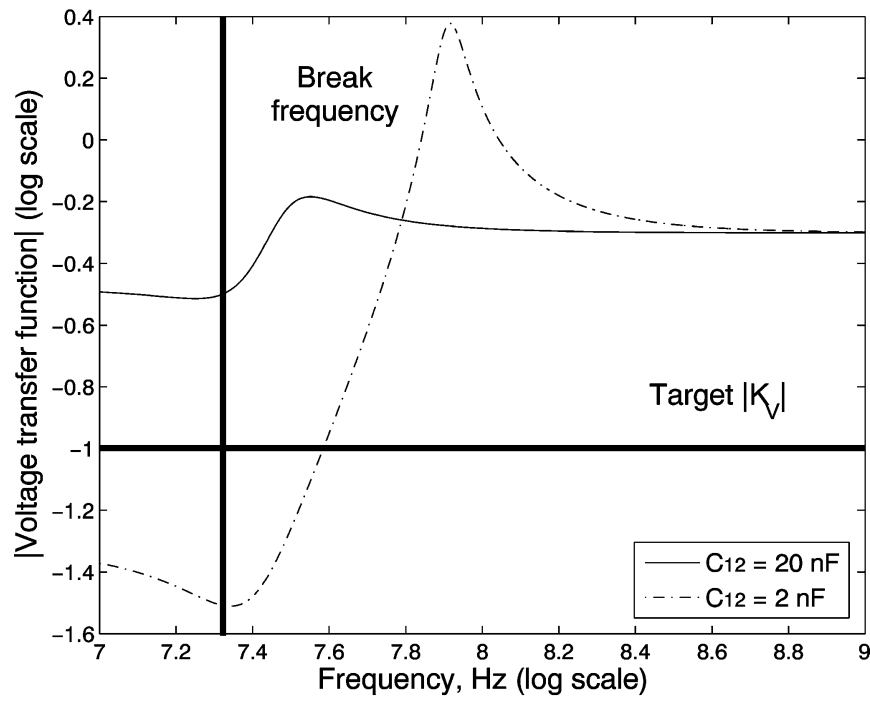

(b)

Fig. 15. Magnitude of the voltage transfer function of an example dual $V_{\mathrm{dd}}$ power distribution system as a function of frequency. The ESR and ESL of the decoupling capacitors are represented by $R_{12}$ and $R_{2}$ and $L_{12}$ and $L_{2}$, respectively. (a) To maintain the magnitude of the voltage transfer function below 0.1 for frequencies greater than the brake frequency, both the ESL of the capacitor placed between the two power supplies and the decoupling capacitor should be increased. $R_{12}=R_{2}=0.1 \Omega, C_{12}=20 \mathrm{nF}, C_{2}=40 \mathrm{nF}$, and $L_{2}=1 \mathrm{nH}$. (b) To decouple the power supplies in frequencies ranging from DC to the brake frequency, both the capacitor placed between the two power supplies and the ESL of all of the decoupling capacitors should be decreased. $R_{12}=R_{2}=0.1 \Omega, C_{2}=40 \mathrm{nF}$, and $L_{12}=L_{2}=1 \mathrm{nH}$.

below the target $\left|K_{V}\right|$ in the frequency range from the break frequency to the highest operating frequency of the system. At the same time, the system becomes underdamped and produces an overshoot as shown in Fig. 15(a). Similarly, by decreasing $C_{12}$, the $C_{12} / C_{2}$ ratio is lowered and the magnitude of the voltage response falls below the target $\left|K_{V}\right|$ in the frequency range from DC to the break frequency. The system becomes underdamped and produces an overshoot as shown in Fig. 15(b).

Three different tradeoff scenarios similar to the case study shown in Fig. 14 are summarized in Table II. The design parameters for each scenario represent typical values of board, 
TABLE II

Tradeoff Between the Magnitude and Frequency Range of the Voltage Response

\begin{tabular}{|c|c|c|c|c|c|}
\hline $\begin{array}{l}\text { Tradeoff } \\
\text { Scenario }\end{array}$ & $\begin{array}{l}\text { Power } \\
\text { Distribution } \\
\text { System }\end{array}$ & Minimum $\left|K_{V}\right|$ & Maximum $\left|K_{V}\right|$ & $\begin{array}{l}\text { Minimum } \\
\text { frequency }\end{array}$ & $\begin{array}{l}\text { Maximum } \\
\text { frequency }\end{array}$ \\
\hline \multirow{3}{*}{1} & Original & 0.30 & 0.50 & $\mathrm{DC}$ & $\infty$ \\
\hline & Increased $L_{12}$ & 0.09 & 0.56 & $63 \mathrm{kHz}$ & $\infty$ \\
\hline & Decreased $C_{12}$ & 0.05 & 0.60 & DC & $63 \mathrm{kHz}$ \\
\hline \multirow{3}{*}{1} & Original & 0.20 & 0.50 & $\mathrm{DC}$ & $\infty$ \\
\hline & Increased $L_{12}$ & 0.09 & 0.50 & $3 \mathrm{MHz}$ & $\infty$ \\
\hline & Decreased $C_{12}$ & 0.03 & 0.60 & DC & $3 \mathrm{MHz}$ \\
\hline \multirow{3}{*}{ III } & Original & 0.20 & 0.50 & $\mathrm{DC}$ & $\infty$ \\
\hline & Increased $L_{12}$ & 0.09 & 0.50 & $3 \mathrm{GHz}$ & \\
\hline & Decreased $C_{12}$ & 0.05 & 0.45 & DC & $3 \mathrm{GHz}$ \\
\hline Scenario I & \multirow{2}{*}{\multicolumn{5}{|c|}{$\begin{array}{l}\text { Original circuit: } R_{12}=R_{2}=2 \mathrm{~m} \Omega, L_{12}=L_{2}=1 \mathrm{nH}, C_{12}=2 \mathrm{mF}, C_{2}=4 \mathrm{mF} \\
\text { Increased } L_{12}: R_{12}=R_{2}=2 \mathrm{~m} \Omega, L_{12}=10 \mathrm{nH}, L_{2}=1 \mathrm{nH}, C_{12}=2 \mathrm{mF}, C_{2}=4 \mathrm{mF} \\
\text { Decreased } C_{12}: R_{12}=R_{2}=2 \mathrm{~m} \Omega, L_{12}=L_{2}=1 \mathrm{nH}, C_{12}=200 \mu \mathrm{F}, C_{2}=4 \mathrm{mF}\end{array}$}} \\
\hline Board & & & & & \\
\hline Scenario II & \multirow{4}{*}{\multicolumn{5}{|c|}{$\begin{array}{l}\text { Original circuit: } R_{12}=R_{2}=10 \mathrm{~m} \Omega, L_{12}=L_{2}=100 \mathrm{pH}, C_{12}=10 \mu \mathrm{F}, C_{2}=40 \mu \mathrm{F} \\
\text { Increased } L_{12}: R_{12}=R_{2}=10 \mathrm{~m} \Omega, L_{12}=1 \mathrm{nH}, L_{2}=100 \mathrm{pH}, C_{12}=10 \mu \mathrm{F}, C_{2}=40 \mu \mathrm{F} \\
\text { Decreased } C_{12}: R_{12}=R_{2}=10 \mathrm{~m} \Omega, L_{12}=L_{2}=100 \mathrm{pH}, C_{12}=1 \mu \mathrm{F}, C_{2}=40 \mu \mathrm{F} \\
\text { Original circuit: } R_{12}=R_{2}=10 \mathrm{~m} \Omega, L_{12}=L_{2}=100 \mathrm{fH}, C_{12}=20 \mathrm{nF}, C_{2}=40 \mathrm{nF} \\
\text { Increased } L_{12}: R_{12}=R_{2}=10 \mathrm{~m} \Omega, L_{12}=1 \mathrm{pH}, L_{2}=100 \mathrm{fH}, C_{12}=20 \mathrm{nF}, C_{2}=40 \mathrm{nF} \\
\text { Decreased } C_{12}: R_{12}=R_{2}=10 \mathrm{~m} \Omega, L_{12}=L_{2}=100 \mathrm{fH}, C_{12}=2 \mathrm{nF}, C_{2}=40 \mathrm{nF}\end{array}$}} \\
\hline Package & & & & & \\
\hline Scenario III & & & & & \\
\hline On-chip & & & & & \\
\hline
\end{tabular}

package, and on-chip decoupling capacitors, as shown in Fig. 9. The original system in each scenario produces an overshoot-free voltage response over a wide range of operating frequencies from DC to the highest operating frequency of the system. By increasing the ESL of the decoupling capacitor placed between the two power supplies, the system produces an overshoot and the range of operating frequencies is divided by two. The same phenomenon takes place if the value of the decoupling capacitor placed between the two power supplies is decreased. In the first case, when the ESL is increased by an order of magnitude, the magnitude of the voltage response is lowered by more than an order of magnitude from the break frequency to infinity. When $C_{12}$ is decreased by an order of magnitude, the magnitude of the voltage response is lowered by more than an order of magnitude from DC to the break frequency. Note from the table that the location of the break point depends upon the particular system parameters. The break frequency of the board system occurs at a lower frequency as compared to the break frequency of the package power delivery network. Similarly, the break frequency of the package power distribution system is lower than the break frequency of the on-chip system. As previously mentioned, for typical power supplies values and allowed ripple voltage, $\left|K_{V}\right|$ should be less than 0.1 to decouple a noisy power supply from a quiet power supply. As listed in Table II, this requirement is satisfied for the power distribution system if $L_{12}$ is increased or $C_{12}$ is decreased. The magnitude of the overshoot falls rapidly with decreasing ESL of the decoupling capacitors. Due to the extremely low value of the ESL in an on-chip power network, typically several hundred femtohenrys, the magnitude of the overshoot does not exceed the maximum magnitude of the overshoot-free voltage response.

Unlike the design methodology for producing an overshootfree response described in Section V-A, a design methodology to tradeoff the magnitude of the voltage response of the power distribution system with the frequency range of an overshootfree response is as follows. Based upon the available decoupling capacitance the decoupling capacitances for each power supply are determined. Depending upon the target frequency range with respect to the break frequency, the ESL of the capacitor placed between the two power supplies and the decoupling capacitors should both be increased (above the break frequency). Otherwise, the capacitor placed between the two power supplies and the ESL of all of the decoupling capacitors should both be decreased (below the break frequency).

\section{CONCLUSION}

It has become common practice to use multiple on-chip power supply voltages to reduce power dissipation without degrading system speed. To maintain the impedance of a power distribution system below a specified impedance, multiple decoupling capacitors are placed at different levels of the power grid hierarchy. The decoupling capacitors are placed both with progressively decreasing values to shift the antiresonance spike beyond the maximum operating frequency, and with increasing ESR to control the damping characteristics. Increasing ESR also broadens the frequency range of the antiresonant spikes, ensuring that the amplitude of the output impedance remains below the target impedance. Another strategy is to limit the magnitude of the antiresonant spikes by reducing the ESL of all of the decoupling capacitors.

To maintain the magnitude of the voltage transfer function below or equal to 0.5, the ESR and ESL of the decoupling capacitors should be carefully chosen. Generally, to maintain an overshoot-free voltage response over an applicable frequency range, the total ESR of the decoupling capacitors should be greater than the two characteristic impedances of the system, represented by (12). To further decouple the power supplies in frequencies ranging from DC to the break frequency, both the capacitor placed between the two power supply voltages and the ESL of all of the decoupling capacitors should be decreased. Conversely, to decouple the power supplies in frequencies ranging from the break frequency to infinity, both the ESL 
of the capacitor placed between the two power supply voltages and the decoupling capacitors should be increased. If it is not possible to design a power distribution system that satisfies the overshoot-free voltage response criterion, the objective of maintaining $\left|K_{V}\right|$ at a required level will not be satisfied. Alternatively, the frequency range of an overshoot-free voltage response can be traded off with the magnitude of the response.

\section{REFERENCES}

[1] A. Chandrakasan, M. Potkonjak, J. Rabaey, and R. W. Brodersen, "HYPER-LP: A system for power minimization using architectural transformations," in Proc. IEEE Int. Conf. Comput.-Aided Des., 1992, pp. 300-303.

[2] A. Chandrakasan, S. Sheng, and R. Brodersen, "Low-power CMOS digital design," IEEE J. Solid-State Circuits, vol. 27, no. 4, pp. 474-484, Apr. 1992.

[3] J. Mermet and W. Nebel, Low Power Design in Deep Submicron Electronics. Norwell, MA: Kluwer, 1997.

[4] A. Chandrakasan, W. J. Bowhill, and F. Fox, Design of High-Performance Microprocessor Circuits. New York: Wiley, 2000.

[5] K. Usami and M. Horowitz, "Clustered voltage scaling technique for low-power design," in Proc. IEEE Int. Workshop Low Power Des., 1995, pp. 3-8.

[6] J.-M. Chang and M. Pedram, "Energy minimization using multiple supply voltages," IEEE Trans. Very Large Scale Integr. (VLSI) Syst., vol. 5, no. 4, pp. 425-435, Dec. 1997.

[7] S. Raje and M. Sarrafzadeh, "Variable voltage scheduling," in Proc. ACM Int. Symp. Low Power Des., Apr. 1995, pp. 9-14.

[8] A. V. Mezhiba and E. G. Friedman, Power Distribution Networks in High Speed Integrated Circuits. Norwell, MA: Kluwer, 2004.

[9] L. D. Smith, R. E. Anderson, D. W. Forehand, T. J. Pelc, and T. Roy, "Power distribution system design methodology and capacitor selection for modern CMOS technology," IEEE Trans. Adv. Packag., vol. 22, no. 3, pp. 284-291, Aug. 1999.

[10] S. R. Nassif and O. Fakhouri, "Technology trends in power-grid-induced noise," in Proc. ACM Int. Workshop Syst. Level Interconnect Prediction, 2002, pp. 55-59.

[11] L. D. Smith, "Packaging and power distribution design considerations for a sun microsystems desktop workstation," in Proc. Elect. Performance Electron. Packag. Conf., 1997, pp. 19-22.

[12] W. D. Becker et al., "Modeling, simulation and measurement of mid-frequency simultaneous switching noise in computer systems," IEEE Trans. Comp., Packag., Manuf. Technol., vol. 21, no. 2, pt. B, pp. 157-163, May 1998.

[13] H. B. Bakoglu, Circuits, Interconnections, and Packaging for VLSI. Reading, MA: Addison-Wesley, 1990.

[14] I. Novak et al., "Distributed matched bypassing for board-level power distribution networks," IEEE Trans. Adv. Packag., vol. 25, no. 2, pp. 230-243, May 2002.

[15] M. Popovich and E. G. Friedman, "Impedance characteristics of decoupling capacitors in multi-power distribution systems," in Proc. IEEE Int. Conf. Electron., Circuits Syst., Dec. 2004, pp. 160-163.

[16] A. V. Mezhiba and E. G. Friedman, "Inductive properties of high-performance power distribution grids," IEEE Trans. Very Large Scale Integr. (VLSI) Syst., vol. 10, no. 6, pp. 762-776, Dec. 2002.

[17] M. Popovich and E. G. Friedman, "Decoupling capacitors for power distribution systems with multiple power supply voltages," in Proc. IEEE SOC Conf., 2004, pp. 331-334.

[18] — , "Noise coupling in multi-voltage power distribution systems with decoupling capacitors," in Proc. IEEE Int. Symp. Circuits Syst., 2005 , vol. I, pp. $620-623$

[19] — , "Noise aware decoupling capacitors for multi-voltage power distribution systems," in Proc. IEEE Int. Symp. Quality Electron. Des., 2005, pp. 334-339.
[20] V. Kursun, S. G. Narendra, V. K. De, and E. G. Friedman, "Analysis of buck converters for on-chip integration with a dual supply voltage microprocessor," IEEE Trans. Very Large Scale Integr. (VLSI) Syst., vol. 11, no. 3, pp. 514-522, Mar. 2003.

[21] C. R. Paul, Analysis of Linear Circuits. New York: McGraw-Hill, 1989.

[22] M. K. Gowan, L. L. Biro, and D. B. Jackson, "Power considerations in the design of the alpha 21264 microprocessor," in Proc. IEEE/ACM Des. Automat. Conf., 1998, pp. 726-731.

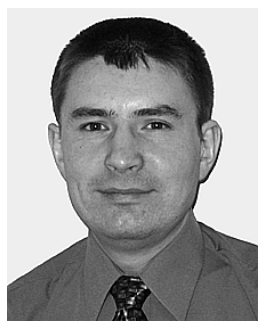

Mikhail Popovich received the B.S. degree in electrical engineering from Izhevsk State Technical University, Izhevsk, Russia, in 1998, and the M.S. degree in electrical and computer engineering from the University of Rochester, Rochester, NY, in 2002. He is currently working toward the Ph.D. degree in electrical and computer engineering at the University of Rochester.

He was an intern at Freescale Semiconductor, Incorporated, Tempe, AZ, in 2005, where he worked on signal integrity in RF and mixed-signal ICs, and also developed design techniques for placing distributed on-chip decoupling capacitors. His research interests are in the areas of noise, signal integrity, and interconnect design, including on-chip inductive effects, optimization of power distribution networks, and the design of on-chip decoupling capacitors.

Mr. Popovich received the Best Student Paper Award at the ACM Great Lake Symposium on VLSI in 2005.

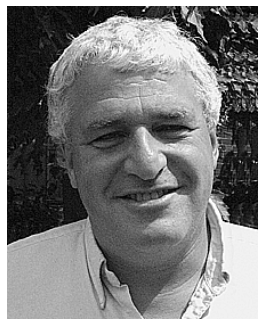

Eby G. Friedman (S'78-M'89-SM'90-F'00) received the B.S. degree from Lafayette College, Easton, PA, in 1979, and the M.S. and Ph.D. degrees from the University of California, Irvine, in 1981 and 1989, respectively, all in electrical engineering.

He has been with the Department of Electrical and Computer Engineering at the University of Rochester, Rochester, NY, since 1991, where he is a Distinguished Professor, the Director of the High Performance VLSI/IC Design and Analysis Laboratory, and the Director of the Center for Electronic Imaging Systems. He is the author of more than 300 papers, book chapters, several patents, and the author or editor of seven books in the fields of high-speed and low-power CMOS design techniques, high-speed interconnect, and the theory and application of synchronous clock and power distribution networks.

Dr. Friedman is a recipient of the Howard Hughes Masters and Doctoral Fellowships, an IBM University Research Award, an Outstanding IEEE Chapter Chairman Award, a University of Rochester College of Engineering Teaching Excellence Award, and the University of Rochester Graduate Teaching Award. $\mathrm{He}$ is a Senior Fulbright Fellow. He was previously the Editor-in-Chief of the IEEE TRANSACTIONS ON VERY LARGE SCALE INTEGRATION (VLSI) SYSTEMS, a Member of the editorial board of the PROCEEDINGS OF THE IEEE and the IEEE TRANSACTIONS ON Circuits AND SYSTEMS II: ANALOG AND Digital Signal PROCESSING, a member of the Circuits and Systems (CAS) Society Board of Governors, CAS liaison to the Solid-State Circuits Society, Chair of the VLSI Systems and Applications CAS Technical Committee, Chair of the Electron Devices Chapter of the IEEE Rochester Section, Program and Technical Chair of several IEEE conferences, and Guest Editor of several special issues in a variety of journals. He is currently the Regional Editor of the Journal of Circuits, Systems and Computers, a Member of the Editorial Boards of Analog Integrated Circuits and Signal Processing, the Microelectronics Journal, and the Journal of VLSI Signal Processing, Chair of the IEEE TRANSACTIONS ON VERY LARGE SCALE INTEGRATION (VLSI) SYSTEMS Steering Committee, and a Member of the Technical Program Committee of a number of conferences. 